\title{
Réussi, atteint, gagné!
}

\section{Christina Aus der Au}

Prof. de théologie, membre de la rédaction Ethique

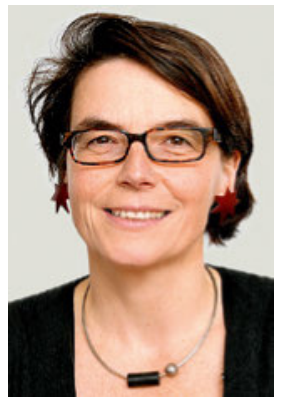

Je suis un peu gênée de l'admettre, mais peut-être ne suis-je pas la seule dans mon cas. Peut-être qu'il y en a d'autres comme moi, qui jouent à des jeux vidéo en passant. Et pas à des jeux cool, ambitieux, où les membres d'une communauté se mesurent les uns aux autres, où l'on doit planifier des dispositifs à long terme, résoudre des énigmes complexes ou trouver des passages dangereux. Pas des jeux où l'on peut comparer fièrement les scores et les niveaux atteints.

Non, des jeux beaucoup simples, sans la moindre prétention intellectuelle, qui ne requièrent même pas une rapidité fulgurante ou une motricité fine exceptionnelle. Je joue au Solitaire ou à Bubbleshooter, à Jewels ou Tetris. Surtout quand je n'arrive pas à avancer sur mon travail. Quand je peine à rédiger un article, qu'un e-mail délicat ne s'écrit pas tout seul ou que je suis en panne d'idées brillantes. Hop, j'ouvre une fenêtre sur le bureau après avoir regardé un peu honteusement autour de moi pour être sûre que personne ne m'observe et mes doigts se mettent à sautiller avec plaisir sur le clavier pour déposer les cartes, les bulles de savon et les blocs au bon endroit. Et à la troisième ou quatrième tentative, quand je réussis enfin, quand les cartes s'ordonnent comme par magie et que les piles disparaissent, quand les dernières bulles d'une même couleur explosent d'elles-mêmes, alors je suis satisfaite. Tout est bien, réglé, résolu.

C'est un sentiment que j'éprouve rarement dans ma vie en dehors des jeux. Quand vient le soir, il reste toujours quelque chose d'inachevé, un truc pas fait, pas assez réfléchi, pas lu ou débattu jusqu'au bout. Et je n'ai même pas encore ouvert le journal! Là, la situation internationale est encore bien plus complexe, inaboutie, insurmontable! Pour chaque problème résolu, dix nouveaux se posent.

Pas étonnant dans ces conditions que j'en revienne toujours à mes cartes et mes bulles de savon! A ces défis à ma portée - et surtout à la satisfaction que me procure la réalisation de l'objectif. Le système de récompense du cerveau est activé, le nucleus accumbens est stimulé... A quand la prochaine satisfaction?

Aristote, le philosophe grec, froncerait les sourcils: «Tu confonds deux types d'activités: il y a d'une part la fabrication, dont l'aboutissement est un produit. Et avoir tiré sur toutes les bulles, rangé toutes les cartes, c'est un produit, au même titre que fabriquer une chaise ou écrire un article. La satisfaction vient lorsque le produit est achevé avec succès - mais le suivant se profile déjà à l'horizon. L'autre sorte d'activité est celle dont la récompense, la gratification réside en elle-même. Celle qui ne satisfait pas seulement à la fin, mais aussi pendant qu'on la pratique. C'est par exemple le cas lorsqu'on fait de la musique ou qu'on lit un ouvrage passionnant. Ou qu'on approfondit une réflexion, étudie, accompagne des gens, exerce son métier en y prenant du plaisir. Tout ce qu'un spécialiste tchèque du bonheur appelle le ‘flow». Les deux types d'activités sont nécessaires, mais pour bien vivre, il ne faut pas seulement sauter de produit en produit, il faut aussi trouver sa paix intérieure.»

Aujourd'hui, près de deux millénaires et demi plus tard, Aristote n'en reviendrait pas de voir tout ce que nous avons transformé en produit! Le succès, c'est d'atteindre un certain échelon de carrière ou niveau de revenus, l'éducation est un diplôme et un papier, la beauté se mesure à l'indice de masse corporelle. Et la santé est obtenue lorsque l'on peut cocher $\mathrm{x}$ pas marchés ou $\mathrm{x}$ bouteilles d'eau bues par jour. Ou tant et tant de calories, de pompes, de tours de stade en courant. Rien d'étonnant à ce que les systèmes de suivi et d'enregistrement de l'activité physique soient si populaires. Ils favorisent le saut de récompense en récompense de la même façon que mes jeux. Réussi, atteint, gagné. Quantifiable d'une petite victoire à la suivante. En soi, il n'y a évidemment rien de mal à cela. Se concentrer sur les petites étapes aide à atteindre les grands objectifs. Mais ces deux aspects restent dans le mode de la réalisation, des produits. Plus les buts sont simples, plus c'est efficace.

Le cerveau est conditionné à recevoir continuellement des récompenses produits. Par petites bouchées, sans arrêt, et à un rythme toujours plus rapide. Et toutes les tâches de longue haleine, qui exigent une immersion soigneuse dans la matière, qui ne donnent pas de résultat immédiat, qui sont complexes ou même insolubles ont perdu d'avance face à cela.

Finalement, je devrais peut-être envisager de supprimer totalement Solitaire et Tetris de mon répertoire. 